\title{
Reconocimiento de emociones a partir de la expresión y el contexto: Una réplica ${ }^{1}$
}

\author{
MARÍA JOSÉ MALLO *, JOSÉ MIGUEL FERNÁNDEZ \\ DOLS * y HARALD WALLBOTT **
}

* Universidad Autónoma de Madrid, * Universidad de Giessen.

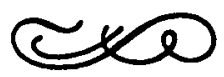

\section{Resumen}

En el presente estudio se examinan las contribuciones relativas de la expresión facial y el contexto situacional al reconocimiento de emociones. Tras la evaluación independiente de ambas fuentes de información, los sujetos juzgaron distintas combinaciones que variaban en su grado de congruencia. Aunque los resultados indican una preponderancia de la información expresiva sobre la situacional en el juicio global, se señala la necesidad de nuevos diseños que permitan investigar el papel modulador del contexto sobre los juicios emocionales.

Palabras clave: contexto, expresión facial, reconocimiento emocional.

\section{Recognition of emotions from expression and context}

\section{Abstract}

The relative contributions of facial expression and situational context on emotions recognition are examined in this study. Both kinds of information were judged alone and after forming combinations with differents degrees of congruency. The results indicate a superiority of expressive cues, bowever more research is needed in order to know the modulation role of context on emotional judgement.

Keywords: context, facial, expression, emotions recognition.

Dirección del autor: Dpto. de Psicología Básica, Social y Metodología. Facultad de Psicología. U.A.M. Cantoblanco (MADRID).

${ }^{1}$ Esta investigación se encuadra en el marco del proyecto PB-85226 de la CAICyT.

* Universidad Autónoma de Madrid.

** Universidad de Giessen. 


\section{INTRODUCCION}

El paradigma clásico utilizado en los estudios de reconocimiento de emociones consiste básicamente en presentar al sujeto un estímulo perteneciente a un determinado canal de expresión, preferentemente el facial y pedirle que elija, dentro de un sistema categorial cerrado, la etiqueta correcta (véase Rosenthal,.1982; Ekman, 1982; Scherer, 1981; Fernández Dols et al., e.p.).

Si bien, algunos estudios introducen variaciones en la medida de la respuesta (dimensiones en lugar de categorías, formatos abiertos) en todos los casos los estímulos presentados son expresiones libres de información contextual. Lógicamente, en las situaciones de la vida real los sujetos difícilmente se enfrentan con una expresión aislada, por el contrario, reciben simultáneamente información sobre el resto de los canales y, sobre el contexto situacional en el que la emoción se expresa.

El primer problema con que se enfrenta la investigación sobre expresión emocional y contexto es la propia definición de este último. De hecho, se trabaja con una definición por defecto: «contexto es todo aquello que no sea expresión», más aún, «contexto es todo aquello que no sea la unidad de expresión que está siendo sometida a análisis». Por ello, se conceptualiza como contexto no sólo los elementos situacionales que acompanan a la expresión, sino también, los otros canales no verbales (voz, movimientos, postura, en relación a la expresión facial) e incluso se habla de contexto intercanal en relación a otras partes de la expresión distintas de la analizada (la expresión facial en su conjunto en relación a la sonrisa) (Wallbott, 1988; Archer y Akert, 1984).

La investigación sobre reconocimiento de emociones a partir de la expresión facial y el contexto situacional ha venido utilizando dos paradigmas clásicos: el denominado paradigma de Goodenough (Goodenough y Tinker, 1931) y el de Munn (1940). El primero de ellos consiste en presentar una expresión facial acompañada por el relato escrito de una situación elicitadora de emoción. El paradigma de Munn es básicamente idéntico, con la excepción de que el contexto se presenta también visualmente, por medio de una fotografía de una escena en la que se ha oscurecido previamente la cara del protagonista de la situación. Este último procedimiento presenta algunas variantes como la utilización de dibujos en el caso de la investigación con niños (Gnepp, 1983) o vídeo-clips (Deutsch, 1974; Wiggers y Van Lieshout, 1985).

Hasta el momento, los resultados obtenidos se caracterizan por su heterogeneidad y la relativa influencia de cada una de estas fuentes en la atribución final continúa siendo una cuestión abierta. Si bien, es cierto que una gran parte de los trabajos (Frijda, 1969; Watson, 1972; Knudsen y Muzeraki, 1983; Reichenbach y Masters, 1983) se decantan por la preponderancia de la expresión facial, son varios los autores que defienden la importancia de las claves contextuales en el reconocimiento de emociones (Goldberg, 1951; Forgas y Brown, 1977; Spignesi y Shor, 1981).

Ekman, Friesen y Ellsworth (1982) en la única revisión realizada hasta la fecha, exceptuando la de Camras (1986) para los trabajos evolutivos, ponen de manifiesto las deficiencias metodológicas de la investigación en el área, fundamentalmente la falta de control sobre los estímulos (expresión y contexto) que se manejan. Señalan la necesidad de valoraciones indepen- 
dientes de fotografía y situación con el fin de hacerlas equivalentes en dimensiones tales como: intensidad, claridad $y$ ambigüedad, factores que pueden interferir en los resultados. De hecho, los autores se inclinan por la mayor fuerza de la expresión en la formación del juicio emocional, pero indican que el contexto puede llegar a ser más importante cuando la expresión sea ambigua o refleje una emoción de baja intensidad.

Wallbott (1988) recogiendo todas estas recomendaciones metodológicas lleva a cabo un experimento cuyo diseño permitió controlar la intensidad y claridad de las fuentes de información presentadas y analizar el modelo de integración de la información utilizado por los sujetos. Siguiendo el modelo de procesamiento de información propuesto por Frijda (Frijda, 1969), Wallbott propone un subprocesamiento separado para la información de la expresión y la del contexto. Como resultado de cada uno de estos subprocesamientos se generan dos «listas» de etiquetas emocionales, donde las emociones están ordenadas de modo decreciente en función de su intensi$\mathrm{dad} /$ probabilidad de atribución al estímulo presentado (cara o contexto). El juicio definitivo del sujeto surgirá de la integración de ambas listas.

Los resultados de su estudio, expuestos en la tabla I, indican, que las correlaciones entre los juicios sobre la expresión facial y la combinación total son mucho más altas que las de esta última con el contexto. Dichos resultados son interpretados en el sentido de una mayor fuerza de la información derivada de la expresión facial en el reconocimiento. El resultado se constata con independencia del tipo de combinación (concordante frente a discordante), aunque el peso del contexto se incrementa en las combinaciones concordantes.

Nuestro propósito en el presente estudio es comprobar la estabilidad de los resultados replicando el experimento de Wallbott, utilizando idéntico diseño y procedimiento.

\section{METODO}

El diseño replicó todas las fases realizadas por Wallbott. Así, un grupo de 15 sujetos (estudiantes de la Facultad de Psicología) evaluó un conjunto de fotografías procedentes de la serie "Pictures of Facial Affect» de Ekman (Ekman y Friesen, 1976). La tarea de los jueces consistía en juzgar la emoción o emociones expresadas por la cara utilizando escalas de 8 puntos $(0$ no emoción-8 emoción muy intensa) para cada una de las 9 etiquetas propuestas (alegría, tristeza, miedo, cólera, asco, sorpresa, vergüenza, escepticismo y desesperación). Con este procedimiento se aseguraba la posibilidad de elección múltiple.

A partir de estos juicios se seleccionaron 11 fotografías, utilizando como criterio que la cara expresara la emoción de forma no compleja y no ambigua, lo que se traduce en juicios de alta intensidad en una sola categoría emocional y juicios de baja intensidad en el resto de las categorías.

Para la selección de los contextos se procedió de modo análogo, un segundo grupo de 15 sujetos juzgó 24 descripciones de situaciones procedentes de un banco de situaciones obtenido a través de un estudio transcultural de cuestionario, en el cual los sujetos relataban situaciones elicitadoras de emoción (Scherer, Wallbott y Summerfield, 1986). Utilizando el mismo criterio de baja ambigüedad y complejidad se seleccionaron 11 descripciones para el experimento. 
A partir de estos dos conjuntos de estímulos se procedió a combinarlos tres veces entre sí de forma aleatoria, obteniéndose un total de 33 combinaciones. De estas 33 combinaciones 11 resultaron concordantes, 11 discordantes y 11 ambiguas. El grado de concordancia de una combinación viene definido por la correlación entre la fotografía y la situación que la componen, correlación cuya media fue de 0,70 para las concordantes, - 0,32 para las discordantes y 0,11 para las ambiguas. La asignación de cada combinación concreta a cada uno de estos grupos no coincidió totalmente con la del Wallbott, debido a que el grado de congruencia se establece en función de las correlaciones previas expresión/contexto y las valoraciones previas de ambas fuentes fueron en algunos casos distintas de las obtenidas en la muestra alemana. En concreto 8 de las 33 combinaciones no fueron asignadas a los mismos niveles en las 2 muestras.

Cada tipo de combinación fue evaluada por un grupo de sujetos en los mismos términos en que se habían realizado las valoraciones aisladas de foto y situación.

El diseño, de modo esquemático, quedaría como sigue:

\section{GRUPO $1 \quad \mathrm{~N}=15$ \\ FOTOGRAFIAS}

$\begin{array}{lccc}\text { GRUPO } 3 & \mathrm{~N}=15 & \text { GRUPO 4 } & \mathrm{N}=15 \\ \text { COMBINACIONES } & \text { COMBINACIONES } \\ \text { CONCORDANTES } & \text { DISCORDANTES }\end{array}$

GRUPO $2 \quad \mathrm{~N}=15$ SITUACIONES

\author{
GRUPO $5 \quad \mathrm{~N}=15$ \\ COMBINACIONES \\ AMBIGUAS
}

\section{RESULTADOS}

Para determinar la influencia relativa de la expresión facial y el contexto sobre el reconocimiento se procedió a correlacionar las puntuaciones de intensidad, en cada categoría emocional para cada combinación, con las puntuaciones obtenidas para la foto integrante de la combinación, y con las obtenidas para la descripción.

Con el fin de averiguar si las diferencias entre los tres tipos de combinación eran estadísticamente significativas se llevó a cabo un ANOVA de un factor cuyos resultados se exponen en la tabla I. Una prueba de Newman-Keuls de comparaciones múltiples mostró entre qué niveles concretos del factor se producían las diferencias significativas. De nuevo, las correlaciones de las combinaciones son mayores con la expresión facial que con el contexto y ello ocurre en los tres tipos de combinaciones. Sin embargo, se producen diferencias significativas en el peso del contexto según el tipo de combinación, aunque siempre dentro de la tónica general de superioridad de la expresión facial. El peso del contexto es mayor en la combinación concordante, que en la ambigua, y mayor en ésta que en la discordante $(\mathrm{F}=34,57 ; \mathrm{p}<.000)$. El ANOVA no resultó, sin embargo, significativo para la correlación entre combinación y fotografía, donde no aparecieron diferencias entre los tres tipos de combinación en relación al comportamiento de la expresión facial $(\mathrm{F}=2,32 ; \mathrm{p}>.05)$.

Se calcularon otras dos medidas que convergen con el resultado obtenido. El índice propuesto por Frijda en los trabajos pioneros en los campos (Frijda, 1969) constituye la medida clásica para averiguar la contribu- 
TABLA I

Resultados obtenidos en el estudio de Wallbott (1988) (pimera linea de datos) y en el presente estudio (segunda linea de datos)

\begin{tabular}{|c|c|c|c|c|c|c|c|c|}
\hline & \multicolumn{3}{|c|}{ Tipo de combinación } & \multirow{2}{*}{ Media } & \multirow[b]{2}{*}{$\mathbf{F}$} & \multirow[b]{2}{*}{ gl } & \multirow[b]{2}{*}{$\mathbf{p}$} & \multirow{2}{*}{$\begin{array}{l}\text { Comparaciones } \\
\text { múltiples } \\
\text { Newman-Keuls }\end{array}$} \\
\hline & $\underset{N=11}{\text { DIS. }}$ & $\begin{array}{l}\mathbf{A M B} \\
\mathbf{N}=11\end{array}$ & $\begin{array}{l}\text { CON. } \\
N=11\end{array}$ & & & & & \\
\hline Correlación media foto/contexto & $\begin{array}{l}-.33 \\
-.32\end{array}$ & $\begin{array}{l}.10 \\
.11\end{array}$ & $\begin{array}{l}.86 \\
.70\end{array}$ & $\begin{array}{l}.21 \\
.16\end{array}$ & $\begin{array}{l}148.93 \\
122.00\end{array}$ & $\begin{array}{l}2 / 30 \\
2 / 30\end{array}$ & $\begin{array}{l}.000 \\
.000\end{array}$ & $\begin{array}{l}\mathrm{CON}>\mathrm{AMB}>\mathrm{DIS} \\
\mathrm{CON}>\mathrm{AMB}>\mathrm{DIS}\end{array}$ \\
\hline Correlación media foto/combinación & $\begin{array}{l}.82 \\
.75\end{array}$ & $\begin{array}{l}.77 \\
.68\end{array}$ & $\begin{array}{l}.94 \\
.89\end{array}$ & $\begin{array}{l}.84 \\
.77\end{array}$ & $\begin{array}{l}3.10 \\
2.32\end{array}$ & $\begin{array}{l}2 / 30 \\
2 / 30\end{array}$ & $\begin{array}{l}>.05 \\
>.05\end{array}$ & \\
\hline Correlación media contexto/combinación & $\begin{array}{r}-.05 \\
.04\end{array}$ & $\begin{array}{l}.49 \\
.58\end{array}$ & $\begin{array}{l}.91 \\
.85\end{array}$ & $\begin{array}{l}.45 \\
.49\end{array}$ & $\begin{array}{l}40.91 \\
34.57\end{array}$ & $\begin{array}{l}2 / 30 \\
2 / 30\end{array}$ & $\begin{array}{l}.000 \\
.000\end{array}$ & $\begin{array}{l}\mathrm{CON}>A M B>D I S \\
C O N>A M B>D I S\end{array}$ \\
\hline Indice de cambio relativo & $\begin{array}{l}.55 \\
.45\end{array}$ & $\begin{array}{l}.59 \\
.81\end{array}$ & $\begin{array}{r}1.05 \\
.87\end{array}$ & $\begin{array}{l}.73 \\
.71\end{array}$ & $\begin{array}{l}4.07 \\
3.34\end{array}$ & $\begin{array}{l}2 / 30 \\
2 / 30\end{array}$ & $\begin{array}{l}.027 \\
.049\end{array}$ & $\begin{array}{l}\mathrm{CON}>A M B=D I S \\
C O N=A M B>D I S\end{array}$ \\
\hline $\begin{array}{l}\text { Pesos beta } \\
\text { foto }\end{array}$ & $\begin{array}{l}.90 \\
.84\end{array}$ & $\begin{array}{l}.78 \\
.61\end{array}$ & $\begin{array}{l}.52 \\
.59\end{array}$ & $\begin{array}{l}.73 \\
.68\end{array}$ & $\begin{array}{l}3.63 \\
2.45\end{array}$ & $\begin{array}{l}2 / 30 \\
2 / 30\end{array}$ & $\begin{array}{l}.039 \\
>.05\end{array}$ & $\mathrm{DIS}>\mathrm{CON}$ \\
\hline $\begin{array}{l}\text { Pesos beta } \\
\text { contexto }\end{array}$ & $\begin{array}{l}.26 \\
.30\end{array}$ & $\begin{array}{l}.41 \\
.54\end{array}$ & $\begin{array}{l}.48 \\
.44\end{array}$ & $\begin{array}{l}.38 \\
.43\end{array}$ & $\begin{array}{l}<1 \\
2.23\end{array}$ & $\begin{array}{l}2 / 30 \\
2 / 30\end{array}$ & $\begin{array}{l}>.05 \\
>.05\end{array}$ & \\
\hline
\end{tabular}


ción relativa de cada una de estas dos fuentes. Se calcula dividiendo el sumatorio de las diferencias absolutas entre los juicios a la combinación y los juicios a la expresión facial por el sumatorio de las diferencias absolutas entre los juicios a la combinación y los juicios al contexto. Si dicho índice es - menor que 1, la información de la expresión facial tiene mayor peso. Como puede observarse en la tabla I, el cálculo del índice de Frijda replica el resultado obtenido.

Otros indicadores en la misma línea fueron los pesos beta obtenidos a partir de la ecuación de regresión múltiple, utilizando como criterio el juicio a la combinación y como variables predictoras los juicios a la expresión facial y a la información contextual. Los resultados, expuestos en el último apartado de la tabla I, indican que los pesos beta para la expresión facial son más altos que para la información derivada del contexto, siendo esta diferencia más acusada para la combinación discordante (.84 vs .30), y disminuyendo en la ambigua (.61 vs .54) y en la consonante (.52 vs .44). A pesar de estas difrencias, el factor tipo de combinación no tuvo efecto estadísticamente significativo ni para la foto $(F=2,45 ; \mathrm{p}>.05)$, ni para el contexto $(F=2,23 ; p>.05)$. Como puede apreciarse los resultados coinciden con los obtenidos por Wallbott en términos de tendencia, aunque en su trabajo la afirmación sobre la superioridad de los pesos beta correspondientes a la foto en la combinación discordante respecto a la concordante, puede hacerse con seguridad estadística.

\section{DISCUSION}

Los resultados obtenidos apuntan en primer lugar a una mayor influencia de la información expresiva en detrimento de la derivada del contexto en la formación de juicios sobre emoción. Esta interpretación se sustenta en los tres índices calculados: correlaciones más altas entre fotografía y combinación, índice de Frijda menor que 1 y mayor cuantía de los pesos beta asociados a la expresión en la ecuación de regresión múltiple. Estos resultados son básicamente coincidentes con los de Wallbott, aunque los valores absolutos difieren ligeramente.

A pesar de la superioridad de la expresión sobre el contexto, la magnitud de las diferencias no se mantiene constante en los tres tipos de combinaciones utilizadas (concordante, ambigua, discordante). El peso del contexto se incrementa en las combinaciones consonantes y se hace mínimo cuando la fotografía y la situación conllevan emociones no relacionadas. Wallbott encontró este mismo resultado en su trabajo y lo interpreta como «una mayor influencia del contexto en las combinaciones consonantes». Esta interpretación puede ser cierta, pero es difícilmente comprobable dado que el sujeto puede haberse basado sólo en una de las fuentes y la alta correlación con ambas continuaría manteniéndose. Para probar esta hipótesis serían necesarios análisis más finos que permitieran comparar el patrón global del juicio ante la combinación con los correspondientes a cada uno de los dos elementos aislados, objetivo sobre el que se está actualmente trabajando.

Podemos concluir, por tanto, que los resultados obtenidos por Wallbott se replican en la muestra española. Sin embargo, a pesar de la estabilidad con que se demuestra la mayor influencia de la expresión, la cuestión 
no queda cerrada. Determinar una mayor influencia de la expresión en el reconocimiento no agota las preguntas sobre el papel del contexto en la atribución de emociones a otros. El principal problema con que se enfrenta la investigación sobre el tema es la carencia de criterios para trabajar sobre el contexto. Carencia que impide la comparación de los diseños y resultados entre los diferentes estudios y, sospechamos, causa de la heterogeneidad de aquéllos. Contextos de diversas temáticas, con diferentes estructuras subyacentes, con diferentes grados de abstracción y complejidad, son manejados en bloque único frente a una expresión facial susceptible de análisis de alta molecularidad. Frente a instrumentos como el FACS (Ekman y Friesen, 1978) y el MAX (Izard, 1979) que permiten la descomposición de la expresión en unidades de acción, no existe ningún sistema taxonómico, ni siquiera remotamente equivalente, para el análisis del contexto.

En definitiva, independientemente de que la expresión facial tenga mayor fuerza que el contexto en el juicio del sujeto, el trabajo debe encaminarse a determinar cómo el contexto puede mediatizar dichos juicios, y lógicamente ello pasa por la investigación previa de las dimensiones que puedan caracterizarlo en términos relevantes al reconocimiento de emociones. En esta línea, podríamos citar el trabajo de uno de los autores (Fernández Dols y Jiménez, 1986) en el que se propone un esquema de caracterización de las situaciones emocionales en términos de cadenas de expectativas y metas más un elemento evaluativo. Dicho esquema supone un primer intento de elaborar un sistema descriptivo de análisis del contexto en función de sus características estructurales.

\section{Referencias}

ARCher, D., y AKerT, R. M. (1984). Problems of context and criterion in nonverbal communication: A new look at the accuracy issue. En M. Cook (ed.): Issues in person perception. Londres: Methuen.

CAMRAS, L. A. (1986). Judgments of emotion from facial expression and situational context. En C. E. Izard y P. B. Read (eds.): Measuring emotion in infants and children. Cambridge: Cambridge University Press.

DEUTSCH, F. (1974). Female preschoolers' perception of affective responses and interpersonal behavior in videotaped episodes. Developmental Psychology 10, 733-740.

EKMan, P. (1982). Emotion in the human face. Cambridge: Cambridge University Press.

Ekman, P., y FrIesEN, W. V. (1976). Pictures of facial effect. Palo Alto, California: Consulting Psychologist Press.

EKman, P., y Friesen, W. V. (1978). The Facial Action Coding System (FACS). Palo Alto, California: Consulting Psychologist Press.

EKMAN, P.; FRIESEN, W. V., y ELLSWORTH, P. (1982). What are the relative contributions of facial behavior and contextual information to the judgment of emotion? En P. Ekman (ed.): Emotion in the human face. Cambridge: Cambridge University Press.

FERNÁNDEZ DOLS, J. M., y JimÉNEZ, A. (1986). The Spanish case: The written expression of emotional routes. En K. R. Scherer, H. G. Wallbott, y A. Summerfield (eds.): Experiencing emotion: A cross-cultural study. Cambridge: Cambridge University Press.

FERNANDEZ Dols, J. M.; IglesiaS, J.; y MaLlo, M. J. (e.p.). Comportamiento no verbal y emoción. En J. Mayor y J. L. Pinillos (eds.): Tratado de Psicología, vol. 3. Madrid: Alhambra.

FORGAS, J. P., y BROWN, L. B. (1977). Environmental and behavioral cues in the perception of social encounters: An exploratory study. American Journal of Psychology 90, 635-644.

FRIJDA, N. A. (1969). Recognition of emotion. En L. Berkowitz (ed.): Advances in Experimental Social Psychology. Nueva York: Academic Press.

GNEPP, J. (1983). Children's social sensitivity: Inferring emotions of conflicting cues. Developmental Psychology 19, 805-814.

GOLDBERG, H. D. (1951). The role of "cutting" in the perception in motion picture. Journal of Applied Psychology 35, 70-71. 
GOODENOUGH, F. L., y TINKER, M. A. (1931). The relative potency of facial expression and verbal description of stimulus in the judgment of emotion. Comparative Psychology 12, 375-370.

IZARD, C. E. (1979). The maximally discriminative facial movement coding system (MAX). Neward: Instructional Resources Center, University of Delaware.

KNUDSEN, H. R., y MUZERAKI, L. H. (1983). The effects of verbal statements of context on facial expressions. Journal of Nonverbal Behavior 7, 202-211.

MUNN, N. L. (1940). The effect of knowledge of situation upon judgment of emotion from facial expressions. Journal of Abnormal and Social Psychology 35, 324-338.

REICHENBACH, L., y MASTERS, J. C. (1983). Children's use of expressive and contextual cues in judgments of emotion. Child Development 54, 993-1004.

ROSENTHAL, R. (1982). Conducting judgment studies. En K. R. Scherer y H. Giles (eds.): Social Markers in Speech. Cambridge: Cambridge University Press.

SCHERER, K. R. (1981). Speech and emotional states. En J. K. Darby (ed.): Speech evaluation in psychiatry. Nueva York: Grune y Stratton.

SCherer, K. R.; Walibott, H. G., y Summerfield, A. B. (eds.) (1986). Experiencing emotion: $A$ cross-cultural study. Cambridge: Cambridge University Press.

SPIGNESI, A., y SHOR, R. E. (1981). The judgment of emotion from facial expressions, contexts and their combination. Journal of General Psychology 104, 41-58.

WALLBOTT, H. G. (1988). Faces in context: The relative importance of facial expression and context information in determinig emotion attributions. En K. R. Scherer (ed.): Facets of emotion. Hillsdale, Nueva York: Lawrence Erlbaum.

Watson, S. G. (1972). Judgment of emotion from facial and contextual cue combinations. Journal of Personality and Social Psychology 24, 332-334.

WIGGERS, M., y VAN LIESHOUT, C. F. (1985). Development of recognition of emotions: Children's reliance on situational and facial expressive cues. Developmental Psychology 21, 338-349. 